\title{
Exploring Public Wearable Display of Wellness Tracker Data
}

\author{
Ashley Colley ${ }^{\mathrm{a}}$, Bastian Pfleging ${ }^{\mathrm{b}, \mathrm{c}}$, Florian Alt ${ }^{\mathrm{d}}$, Jonna Häkkilä ${ }^{\mathrm{a}}$ \\ ${ }^{a}$ University of Lapland, Finland \\ ${ }^{b}$ Eindhoven University of Technology, The Netherlands \\ ${ }^{c} L M U$ Munich, Germany \\ ${ }^{d}$ Bundeswehr University Munich, Germany
}

\begin{abstract}
We investigate wearable presentation of tracked wellness data, and people's perceptions and motivations for sharing it through a wearable display. Whilst online sharing is a common feature in wellness tracking solutions, the motivations and experiences of users to share tracked data in situ has not been widely studied. We created two functional prototypes - the hat tracker and the tracker badge - which we used as probes in two focus groups to elicit opinions on the content and format of wearable tracker displays. Complementing this, a study where participants used the hat tracker prototype in public locations provides insights on sharing in everyday life use contexts. We report that users appreciate the motivating nature of such displays, but favor the display of positive information. Leveraging prior work, we present a model describing the factors affecting users' willingness to share tracked data via wearable displays, and highlight such displays' potential for supporting behavior change.
\end{abstract}

\section{Introduction}

During the past few years, personal wellness and activity tracking has 3 become a common trend and commercial solutions ranging from bracelets 4 to rings and garment-integrated activity trackers are available at affordable 5 prices. Interest in wellness tracking has expanded from the original small 6 group of quantified-self users $[1,2]$ to cover broader user groups. Whereas 7 early activity tracking products focused on heartbeat, routes and step count8 ing, the variety of tracking applications has now expanded to cover areas that 


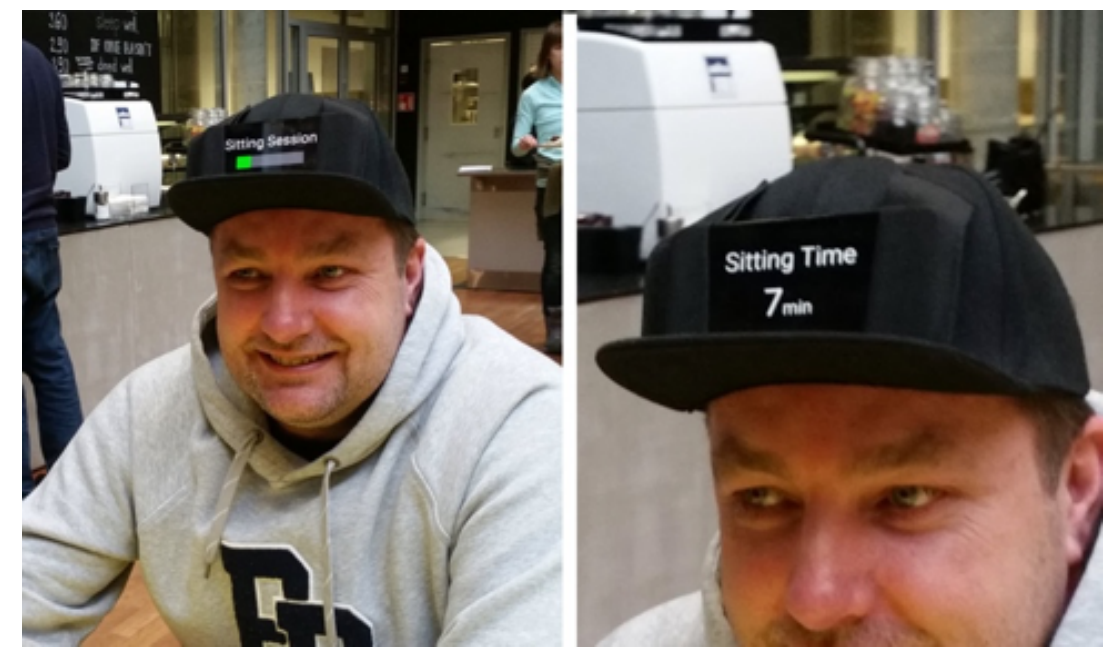

Figure 1: The Hat Tracker allowed us to investigate factors affecting users' willingness to share tracked data.

are more challenging and combine several data sources to infer the actual activities being undertaken. Examples of more complex activity trackers are sleep trackers and holistic wellness applications, which fuse together information from several sources, including physiological or contextual measurements, for instance heartbeat, oxygen saturation, body temperature, movement, and altitude. This development means that we have ever more personal tracking data available from different aspects of our lives.

Ubiquitous connectivity and the wide penetration of social media has made sharing personal tracker data easy. Many activity tracker Apps and platforms, such as Sportstracker, RunTastic, and Endomondo ${ }^{1}$, support sharing workout results online. Sharing sports performance has been reported to have positive effects on motivation, due to peer support and competitiveness $[3,4,5,6,7]$. However, thinking beyond the domain of sport, i.e., the wider scope of health and wellness, it is interesting to examine the sensitivity of different data types, and users' willingness to share them, particularly as available data is becoming increasingly descriptive. Moreover, new methods of sharing are now possible, for instance through wearable displays, such as shirts displaying the wearer's heart rate or real-time jogging perfor-

\footnotetext{
${ }^{1}$ https://www. sports-tracker.com, https://www.runtastic.com, https://www . endomondo.com, last access: 2019-11-17
} 
mance $[8,9]$. With these new wearable form factors, people can share their tracker data in public with others in their physical location.

We examine issues surrounding the use of wearable displays to present wellness tracker data. To provoke discourse, we created two functional wearable display trackers (see Figures 1-3). We used these prototypes as probes in two focus groups and evaluated the hat tracker in an in-the-wild user study. The results shed light on how wearable public displays are perceived, how they can motivate users, and what role the perception of others plays.

Based on the findings from these user studies and an in-depth discussion, we propose a framework identifying the parameters governing users' motivation and willingness to utilize wearable tracker data displays.

The contribution of this work is threefold:

- Two functional prototype wearable displays, visualizing tracker data.

- Findings from two focus groups and an in-the-wild study on the concept of wearable display of tracker data.

- A framework describing the key parameters impacting wearable display of tracker data.

This paper is structured as follows: 1) We present an overview of the related work, focusing on wellness data sharing and particularly on works that have explored wearable display of personal data in different forms. 2) We describe the design and functionality of our two functional wearable tracker display prototypes. 3) We present findings from focus groups and in-thewild evaluations. 4) We critically discuss the findings from our studies, and highlight future directions. 5) Based on our findings we introduce a framework for the wearable display of tracker data.

\section{Related Work}

Today, commercial devices and applications allowing wellness and activity data to be collected and shared online are available to mass markets. These devices track not only activity data, based on motion sensors and GPS, but also a vast amount of physiological data such as heart rate, skin conductance, etc. Utilising sensor fusion and long-term data, such systems are able to make inferences about deeper health and wellness issues, such as for instance sleep problems and over-training. 
The process of sharing personal activity data became easier with the introduction of mobile phone integrated wellness tracking functions [10], and has since expanded to encompass a wide set of technologies. In a study of activity tracker users [11] 36\% shared their data online, whilst a larger $72 \%$ reported sharing their data verbally, to those around them. To position our work, we review works exploring tracker data sharing, wearable and tangible data displays, and perceived challenges with sharing tracker data.

\subsection{Sharing Activity and Physiological Tracker Data}

\subsubsection{Individual Connectivity}

Sharing physiological data has been suggested as a means to create a feeling of connectivity between individuals. For example, Kim et al. presented BreathingFrame, a photo frame that inflates based on a remote person's breathing, supporting connectedness and stimulating curiosity [12]. Min and Nam [13] and Slovak et al. [14] presented concepts sharing heartbeat and breathing rhythm to another person, creating a sense of connectivity between the partners. United-pulse presents a pair of rings which share the wearer's heartbeat with their partner, experienced as haptic pulses [15].

\subsubsection{Group Connectivity}

With the aim of increasing group bonding, tracked data sharing in outdoor sports contexts are discussed by Fedsov et al. [16] and Wozniak et al. [17]. Fedsov et al. noted that sharing happens not just during the activity, but also pre- and post-activity, with different content being shared at each point [16]. The majority of prior work on the topic has reported that beneficial sharing happens only with like-minded others, i.e. within a group engaging in the same activity at a similar level $[17,18,19,20]$. Sharing with such groups leads to improved performance [21] and long-term engagement [19]. In their stickers for steps - which encourages face-to-face comparing of activity data recorded by a mobile app - the authors' report that the intervention triggered general discussions on activity and wellness [22].

Although sharing to broader groups has been investigated, e.g. leveraging a person's existing social network for motivation [23, 24], there is mixed evidence on its benefit. Fritz et al. [18] found that sharing data with real-life friends and family was generally not motivational. However, the desire to offer encouragement and support to others, based on visibility of their data, has been identified $[18,20]$. Overall prior work has presented mixed findings on the effectiveness of sharing tracker data as a driver for behavior change, 
with the differences being generally attributable to context or baseline of comparison $[3,4,5,6]$.

\subsubsection{Cultural and Gender Influences}

Prior works have highlighted cultural differences in interest to adopt wearable technologies [25, 26]. For example, Wang et al. [25] noted that elderly Chinese and Korean participants exhibited higher interest in using technology to connect with others than their US counterparts. In a study comparing cultural and gender perceptions of wearable display of information on emotional states, conducted in France and Japan, display of information for general social purposes was universally disliked, and more strongly so by female participants [26]. Whilst French participants wished to be fully in control of the publicly displayed information, Japanese participants were accepting of some level of AI based content selection. In a study examining willingness to share health information on social media, Li et al. [27] report that Chinese participants were less willing to seek or share information than Italians. The authors infer that, for the Chinese, this reluctance is based on the perceived risk of being misinformed by non-healthcare professionals.

\subsection{Wearable Displays}

Today, wearables, such as activity trackers, have been widely adopted for data collection [28] and, in addition, have have been presented as output devices in different forms. The design space dimensions for such wearable displays has been explored by various researchers [9, 29, 30]. Important dimensions relate to the targeted viewer of the display (i.e. the wearer or a third party observer), the content, and the source of information, which could either display information provided by the wearer (e.g., personal information), the viewer, or the environment $[9,30]$. In addition, technology-related aspects such as size, shape, orientation, body position, and display technology are of interest [9]. Possible form factors include a brooch, e.g., Bubblebadge [30], otherwise attached to the body [31], or a handbag [32].

Typically, in the case of wellness and physiological data tracking wearables, the output is targeted to the wearer, although it is generally not explicitly hidden from other viewers. The glancability of such displays for the wearer has been explored, e.g. by Gouveia et al. [33]. However, a wide variety of works have investigated wearable public display of tracker data [34, 8, 35]. The open heart cycle helmet [34] uses the wearer's heart rate and displays it to the following cyclists. Similarly, a wearable display shirt worn by one 
runner in a group, and visible to other runners, is presented by Mauriello et al. [8], reporting the display shirt improved group cohesion and motivation. In Activmon, Burns et al. [35] evaluate a wearable activity display, comparing the display of both own and group data.

As well as displaying the wearer's own data, wearable displays can display data from others or from environmental sources [36, 37]. Williams et al. [36] explored a scarf to either display the wearer's own emotional state or the mood of others. Ashford experimented with pendants to visualize the wearer's attention and meditation to others and a barometric skirt that visualizes environment parameters (temperature, pressure and altitude) and their effect on the wearer's body temperature [37].

While there has been a large variety of wearable display concepts presenting activity and physiological data, these have so far been isolated solutions and no structured approach to understanding the issues affecting the design of the display and its perception have been presented.

\subsection{In Situ Display of Tracker Data}

As well as sharing via online and wearable display channels, prior work has considered sharing personal tracker data via fixed public displays or as tangible artefacts. In a recent work, Altmeyer et al. used a fixed public display and semi-anonymous nicknames to display users activity data [38]. They noted that the public display significantly increased step counts and motivation to walk, but raised concerns about the possibility of being confronted by others about performance [38]. Khot and Mueller demonstrated the construction of a physical representation of activity data [39], enabling tangible sharing, and Stusak et al. explored how Activity Sculptures impact running activity [40].

\subsection{Ambient Display of Tracker Data}

As an alternative to explicit, for example, numerical, display of tracker data there has been much work on the use of ambient displays, that require some level of interpretation by the viewer. Aesthetically inspired public sharing of tracked data is demonstrated by Arroyo et al.'s water fountain display [41], Khot et al.'s Tastybeats [42] and Fan et al.'s informative art display [43]. Ambient visualization of tracker data, predominantly with the aim to increase physical activity, has been presented by Davis et al. [44], Nakajima and Lehdonvirta [45], Fortmann et al. [46], and Rogers et al. in their twinkly lights concept [47]. 


\subsection{Concerns on Sharing Tracker Data}

Although many works have reported positive aspects of tracker data sharing, almost all also mention people's concerns about sharing such data. For example whilst noting motivational benefits, Gui et al. [24] and Newman et al. [48] report on users' concerns about their online image and the need to maintain a positive impression of one's self to the social community. Similar issues were found by Hassib et al. [49] who studied users' willingness to share physiological, emotional, and cognitive personal data, finding that the parameter value or valence affects willingness to share [49].

Early work on sharing personal data by Consolvo et al. [50], reflected on users' willingness to share location information, concluding that who, what and why are the criteria applied when selecting to share restricted information. Prasad et al. [51] report that people are less willing to share personal demographic information than information collected by the device. This finding is echoed by Shirazi et al. [52], who report interest in sharing sleep information to selected individuals via social networks, but concern that the information would leak to others. Sharing of heart rate values has been explored in the context of a chat application in the HeartChat app [53]. In a medical context, Jacobs et al. [54] report on cancer patients' reluctance to share information with family and social networks, not wishing to be labeled by their condition. Additionally, whilst patients were willing to share quantitative data with healthcare providers, some reluctance was noted related to subjective information, such as feelings of loneliness [54].

\subsection{Summary}

From prior research, we learn that a huge variety of approaches to sharing wellness and physiological data have been investigated. In some works, the findings have suggested that the act of sharing has been motivational, although in general the motivational effects do not appear to be significant or effective in practice. Underlying these prior findings is the sense that the issue is complex and multi-faceted, with seemingly small details, such as the actual value being shared, the people that it is being shared with, or the presentation approach impacting perception. Additionally, attitudes to public data sharing have been shown to be influenced by culture and gender. With this background, we aim toward the creation of a model, detailing the main parameters of the space. To provide further data, in addition to that provided by prior work, we first create and evaluate two prototype forms of wearable tracker displays and use them as probes in user studies. 


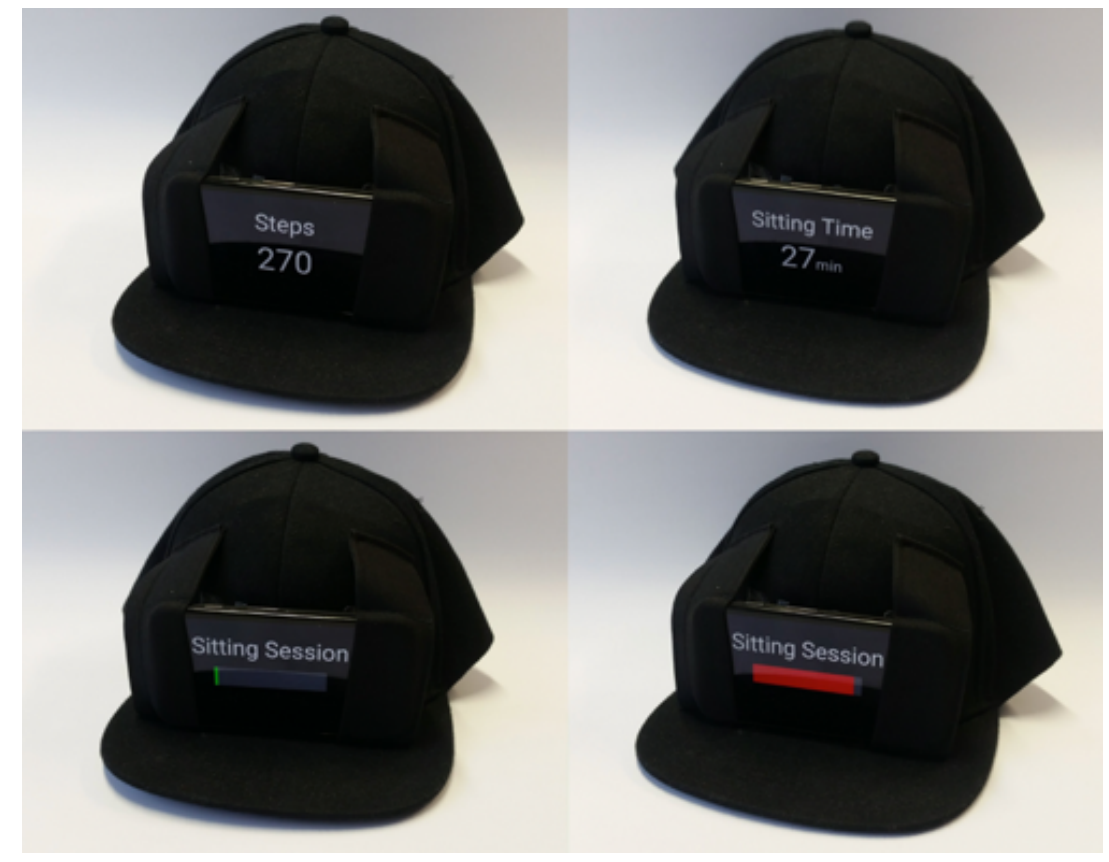

Figure 2: Hat Tracker Prototype, showing the 3 different display modes supported - step count (top left), total sitting time (top right), sitting time bar chart (bottom). The display mode was selected at random every minute.

\section{Prototypes}

To enable the elicitation of user perceptions on the topic of wearable tracker displays, we first created two functional wearable tracker display prototypes: the Hat Tracker and the Tracker Badge.

\subsection{Hat Tracker Prototype}

The Hat Tracker consists of a small Android smartphone (Moto G version 1), securely attached to a baseball cap by an elasticated pouch (Figure 2). As the smartphone was situated on the wearer's head, the platform integrated step count function did not return reliable results. Hence, we developed an Android application, which, based on the output from the smartphone accelerometer, classify each minute of wear as either being sitting or walking. The parameters required for the classification algorithm were established through a short practical iteration process. As step count is nowadays a ubiquitous way of measuring activity, we made a crude translation, converting each active minute to between 70 and 120 steps, depending on the 


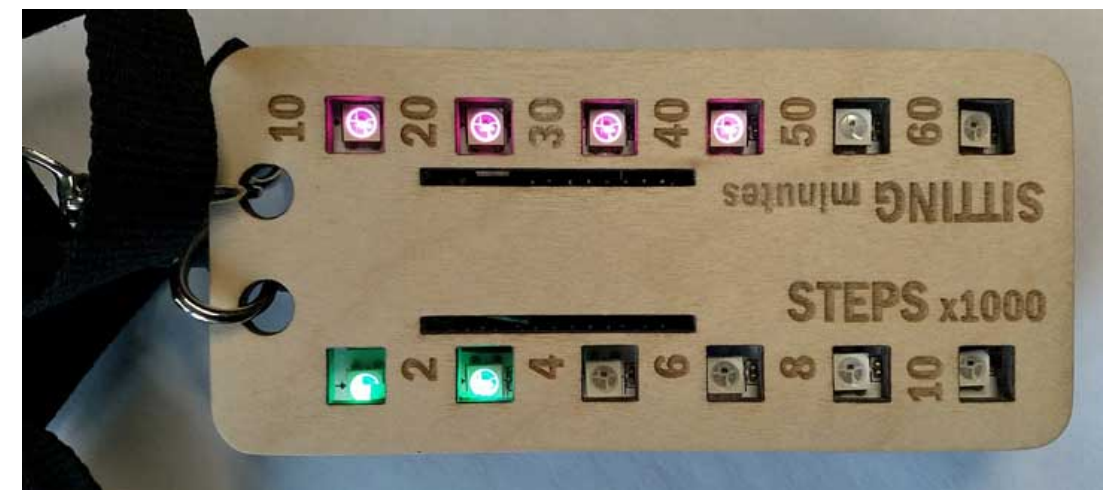

Figure 3: Tracker Badge Prototype, displaying steps and sitting time using LEDs.

amount of motion measured. Informed by the work of Hassib et al. [49] on the impact of data valence on the willingness to share, we selected to present the same data in both polarities, i.e. walking and sitting. The activity status is displayed on the device screen using large numeric visualizations, that bystanders can read at a distance. For sitting time, we utilized a bar chart type visualization. The data displayed on the hat updates once a minute, with the visualization being randomly selected as one of:

- Number of steps, e.g., Steps: 345

- Total sitting time, e.g., Sitting Time: 18 min

- Sitting time bar chart, a green or red colored bar depending on the sitting time value

Whilst the prototype in its current form is clearly not yet suitable for daily wear, we envisaged that its provocative form would be effective in eliciting a wide range of responses from study participants wearing the prototype.

\subsection{Tracker Badge Prototype}

To explore a different wearable form factor and visualization format compared to that of the Hat Tracker, we created the Tracker Badge (Figure 3). As with the Hat Tracker, to expose any differences caused by presentation polarity, the design of the badge aimed to present data in both positive and negative polarities, i.e. sitting and walking. The badge consists of 2 rows of 6 multicolor LEDs, the upper row indicating step count and the lower sitting time. For the step count, each LED illuminated in green represents 2000 
steps, and for the sitting time, each red illuminated LED represents 10 minutes of sitting time. The sitting time count records the time of the current sitting session, and is reset to zero when more than 10 steps are taken within a one minute time window. The display LEDs are driven by a small Arduino micro-controller with on-board Bluetooth low energy connectivity.

All the elements plus a small battery are housed in a conference badge style pouch with a lanyard enabling the badge to be worn around the neck. The data for the Tracker Badge is provided by an Android app, utilizing the platform step count functionality. Once connected to the badge via Bluetooth, the app can run as a service in the background enabling, e.g., the smartphone to be placed in the user's pocket. To enable evaluation of the prototype in a short time, the smartphone app includes settings where the number of steps and sitting time can be multiplied by 10 or 100 times.

\section{Focus Group}

To find insights on the area of wearable tracker data displays, we conducted two focus group-based user studies.

\subsection{Method}

We structured the focus group sessions to investigate participants' views and ideas on wearable display of tracker data, with particular emphasis on what information is displayed, how it is displayed and in what context it is visible (i.e., who sees the information). A moderator guided the discussion towards these topics, supported by probe images. In addition, the focus group participants used our functional prototype wearable tracker displays during the session, such that they would gain insights into displaying their own personal data. The focus group was organised according to the institution's ethical guidelines. As the study did not include children or vulnerable groups, no additional ethics approval was required.

\subsection{Procedure}

The focus groups were run by a single moderator, who also made audio recordings of the sessions which we later transcribed for analysis. We used several different probes to support the participants' discussion and ideation:

- To open the topic of self-tracking, the moderator presented a collection of 8 screenshots from popular smartphone apps as probes. The images 

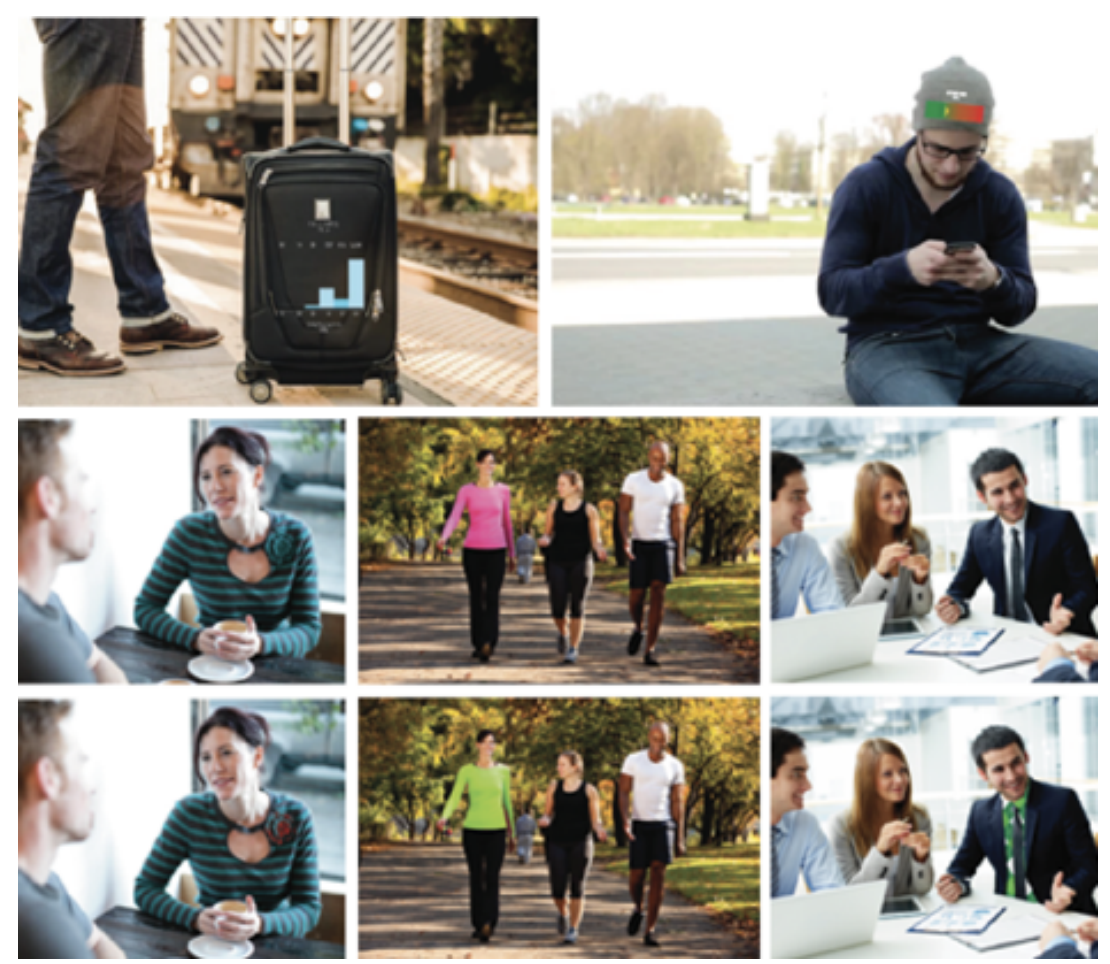

Figure 4: Ideation probe images showing a variety of wearable tracking data display formats in various contexts. Top row: suitcase display at a railway station, hat display in a park. Lower section (showing 2 states of the display in each case): Embroidered color changing badge on left shoulder in a cafe, color changing sports shirt - jogging in a park, pattern changing formal shirt in a business meeting.

were chosen to represent the variety of data sources in tracker apps, e.g., daily steps, amount of sleep, heart rate, performance vs. activity target, etc., as well as the different data visualization approaches used.

- The hat tracker and tracker badge prototypes were introduced and worn by each of the participants for some time during the session.

- A set of images illustrated different contexts and data visualization approaches (Figure 4). These included a column chart on a suitcase, a bar chart on a woolen hat, an embroidered pattern on a shirt that changed color, a sports shirt that changed color, and a formal shirt with a changing pattern. Participants were guided that the information displayed could be various kinds of tracked data. 
- A set of images illustrating different data visualization techniques, e.g., numeric, line charts, bar charts, etc.

During the focus group, the moderator directed discussions to consider: 1) The effect of the data source on their willingness to share such information content about them-selves; 2) If their perception of the value of the data vs. socially accepted values would have any impact on their willingness to share it (for example, step count in respect to a generally known daily target of 10000 steps for healthy living [55]).

\subsection{Analysis}

The sessions lasted approximately 45 minutes each. Altogether 6 participants from different fields (4 female, age 20-27 years) were recruited from the university's test participant pool, and divided into two groups of three (Figure 5). A second researcher (not the moderator) analyzed the transcription from the focus group, who used an affinity grouping approach to identify major themes raised by participants. We identified four themes: the preference for presenting a positive image, the role of social pressure, the influence of the data source, and the format in which the data is presented.

\subsection{Results \\ 4.4.1. Presenting a Positive Image}

The participants' willingness to share data varied, ranging from "Why not, what bad would it [showing data publicly] do?" (\#1) to "I think it would be uncomfortable" (\#2), but group discussions revealed deeper insight to the underlying factors. Participants argued that they were much more willing to publicly display information that presented a positive image of themselves: "I would of course rather show positive [data]! Like look how much I have been walking today!" (\#1). "This one [shirt changing color when steps goal achieved] I could really use, because it has a positive flavor." (\#1). "Would you like to share information that everyone can see: well, he doesn't walk enough? It's like you share negative data about yourself. What you can interpret is that that guy doesn't workout enough or he doesn't take care of his fitness. I wouldn't feel that it would be nice" (\#5). "It [negative information] would easily put a stigma on you. For instance if one only wants to sit in peace for a while, others would think like ha-ha, that guy is just sitting there all the time" (\#4). 
The perception by others and the use context were strongly affecting on the willingness to display data. Altogether, creating a good image of oneself was perceived as very important, e.g., "I feel that this kind of Am-I-incontrol-of-my-life application would be just horrible! For myself I could show [information like this], but not to others. Especially things like, here are my different life sectors, and here I have scored only 4\%" (\#2). Giving a good impression was also commented to be more important in certain situations, or with certain people, than others, e.g., "If you were on your first date and didn't know the other person, it would be awful if you give the impression, like, that guy doesn't do anything else but sit all day long" (\#4).

Interestingly, displaying negative information was seen also as a way to get pity or as an excuse for something; bad mood, tiredness, or wanting to get a seat in the rush hour. It was then seen as a way to show evidence of one's condition: "At the airport that would be useful, because you could justify to someone why they should give you a chair to sleep on, like, look at this, I have slept so little!" (\#3). "To get points for pity. Look how dreadfully hard travel I have had" (\#2). It was commented that the impressions the other would get would change their attitude or behavior towards you: "if I had slept only for 3 hours, they could say at work] Don't take this hard task today, here is an easier one" ( \#2).

\subsubsection{Social Pressure}

Relating to the perception by co-located people, a public wearable display for sharing activity tracking information was seen to create social pressure. There were several comments where participants indicated that creating a good image of themselves would push them to be more active, and pay more attention to it. For example: "[displaying the sitting time on the hat] would at least increase the social pressure. Because everyone else can see how long you have been sitting for" (\#3). "If you feel that sitting is negative, you would start paying more attention to that. Because you wouldn't like to signal to others that you have been sitting a lot." (\#6). Especially, trying on the hat tracker prototype provoked comments in this direction: "This would make me feel anxious, as others can see how much you have been sitting and so" ( \#1, when trying on the hat prototype). "I'd start feeling that I would change my behavior because of this, because you don't want to show that you have been sitting so long and you would somehow like to present a positive image of yourself, like I am a healthy person" (\#5, when trying on the hat prototype). Social pressure through wearable displays could push more than one per- 
son to be more active. The possibility for facilitating healthy group behaviour and gamification, e.g., between colleagues was highlighted: "If all employees had the same display, this could trigger a group exercise break" (\#5).

\subsubsection{Type of Data}

The attitude towards sharing data depended on the type of data, and was linked with the perceived privacy. Sharing activity (steps) was easier to accept than sleeping data. Sleep was perceived as more personal, telling others more about personal life, and the accuracy of sleep data was doubted. E.g.: "It wouldn't hurt to show I have been running this much." (\#5) "Maybe I could display some running, if I would run more often - then I could show my progress. But I don't know about the sleep, if I would share it. Somehow I feel I would get lots of pressure [to sleep well] from that" (\#4). "I don't know if it [displaying sleep time] would cause such [thoughts] as oookay, that guy has been sleeping only three hours. He's been partying yesterday and now he has a hangover!" (\#2).

\subsubsection{Data Presentation}

Data presentation comments related to two aspects: the information visualization style and the wearable form factor. Ease of use and glanceability were appreciated, and for instance displays on sleeves (\#2) and shoes (\#2, \#3) were suggested for jogging. It was perceived that a wearable display would be easier and faster to use than a phone or smartwatch, and would also be more noticeable. Participants commented: "On the sleeves, although it is similar to a watch, [it would be easier to use and see] even if you are wearing a thick coat [and can't easily look at your watch]" (\#2). "It would change [my behavior] because you would simply pay more attention to that. Like if it [wearable display] was green and so on. It would show so clearly that you have been sitting too much and now you need to do something" (\#4). "I think it would be easier than checking the phone all the time to see if you have run enough." (\#6). Thus, if in vicinity, wearable displays were commented to be a good solution for the users themselves.

Comments on the data presentation format highlighted two main themes. Firstly, participants pointed out many times that an abstract, ambient style, with fewer details, would be preferred, as it revealed less information to others, whereas the users themselves would understand the meaning. For instance, "I like that [color changing shirt] much more [than number display]. Because others don't necessarily know that your shirt is changing color, but to 


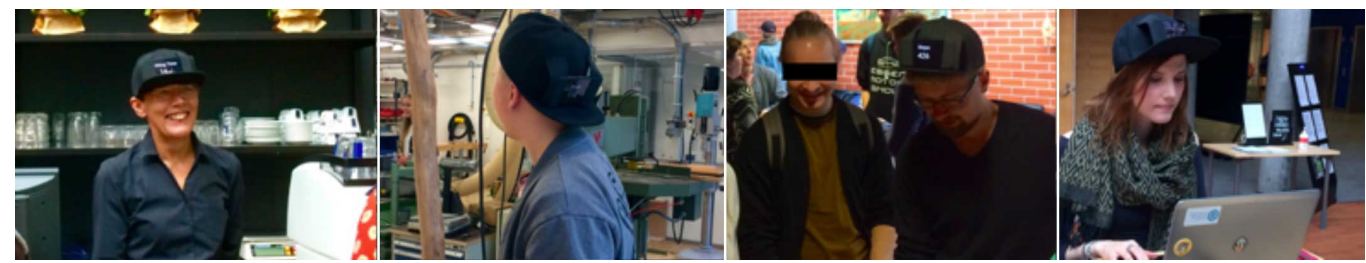

Figure 5: Focus group\#1 discussing around the probe images. Note the participant on the left wearing the Hat Tracker functional prototype.

you, it is like saying: Hah! I have done my jogging for today!" (\#2). Secondly, possible misinterpretation of the data, or one's intentions, provoked concerns. "If I saw that someone's shirt is changing its color, I would think it would relate to temperature or something like that. Then you would think that the wearer is nervous, or sweating, or [I would wonder] what is going on" (\#2).

\section{Field Study}

To complement the focus group and get insights on practical use of public wearable wellness displays, we conducted an additional field study where participants had to wear the hat prototype.

\subsection{Participants and Procedure}

To elicit insights on using public wearable displays, we ran a study requiring participants to wear the hat in public for a short time (30 to 90 minutes) whilst performing their normal daily tasks. We recruited a test group of 12 participants from the university's staff and students. Whilst there were no particular selection criteria, we aimed to recruit a gender and age balanced set of subjects. The study was organised according to the institution's ethical guidelines. As the study did not include children or vulnerable groups, no additional ethics approval was required.

The 12 participants were aged from $21-60$ years $(M=37$ years $)$ and seven were female. Considering prior tracker experience, five had no experience of activity trackers and nine had never tried a sleep tracker. Only one participant was currently using a tracker, to track activity. Of the participants $3 / 12$ stated they were not getting enough exercise and 5/12 not enough sleep. We acknowledge that the prototype tracker displays we created were rather unaesthetic and that our study participants only wore the displays for a short 
time. However, as the displays were fully functional, we believe they enabled participants to quickly feel the issues related to displaying their own data.

\subsection{Results}

Two-thirds of our participants $(67 \%)$ stated that the main reasons they would share wellness tracked data would be self-motivation or to start a discussion with others. Younger members of the study group highlighted competition as one reason to share the data, resulting in overall $42 \%$ of participants mentioning this driver. The perceived effect of data valence was not strong, with only $17 \%$ stated that they would only share positive results. Participants' main reasons for not sharing data were that no one would be interested (57\%) and that they considered the data too private (50\%).

Initially several of our participants were reluctant to wear the hat in public. Although all did, several noted that it caused some social discomfort, e.g., "People were asking and laughing when I had the hat on!" (Female, 59). Overall, participants gave neutral feedback on their opinion of the hat's ability to motivate them to be more active $(M=4.2, S D=1.5$; scale: $1=$ not at all motivational, $7=$ very motivational). We asked participants if they saw a benefit in seeing similar wellness data of those around them. Here, $58 \%$ felt such knowledge would help them better relate to others and be more sympathetic. In this respect participants stating, "As a lecturer I would know if my students have slept enough etc. Would be good to know!" (Male, 40) "I would encourage people to sleep more and be more active, if I'd see them do poorly." (Female, 59)

\subsubsection{Data Source and Valence}

The majority of participants $(92 \%)$ did not consider step count or sitting time to be particularly private information. In contrast around half felt that resting heart rate (58\%) and blood sugar level (50\%) were examples of private information they would not share on a public wearable display. Although in this respect not all agreed, "Depends on the situation. People would know that if my blood sugar level is low, I'm hungry. They would believe me, so it would be like a proof to them." (Female, 28). When asked what other information they would not share on a wearable display $44 \%$ mentioned sleep quality/duration. Interestingly, and rather a dystopian thought, 25\% suggested they would not like any diseases they have to be shown on a wearable display. Participants were reluctant to consider showing negative values on a wearable display, with $50 \%$ stating they would only show positive values. 
One participant stated she would not use the application at all, "If I hide the negative results, then everybody would know why, so I wouldn't even use the whole application." (Female, 26).

\subsubsection{Display Form Factor and Presentation Format}

None of the participants favored a hat or the head area as the location for a wearable display. The most popular form factors/locations stated were accessories (58\%) such and jewelry, a watch shoes and belts. Generally, these suggestions undermine the design target of public visibility, as stated by one participant, "It would be nice to hide the tracker display if necessary." (Female, 28). An interesting pivot for future work was raised by one participant, "I would like to see the information also myself!" (Male, 24). Participants mentioned a variety of preferred visualization formats including numeric values $(33 \%)$, symbols $(33 \%)$, colors and patterns $(42 \%)$ and graphs and bars (42\%). However, there was excitement about the idea of colors and patterns changing, "We need that kind of clothes here that when I have run enough, my co-workers would see it from my clothes via a color change and then I could go and rest for a while." (Female, 59, Restaurant Worker). "You could choose your own theme and an icon! A greyhound for dog-lovers, if you're really active." (Male, 60).

\section{Towards a Model for Wearable Display of Wellness Tracker Data}

Based on our review of the related work, we identified the following factors as contributing to the user experience of using a wearable display showing personal tracker data: the data source, the valence of the data, the presentation form factor and data visualization, and the context of use. To provide a structured view to the topic, we present a model, highlighting the scope and interplay between the various factors (Figure 6).

The value and importance of providing models or frameworks and mapping design spaces has been recognized by the HCI community. They help to not only understand the consequences arising from changes in the design but also help to find and propose new opportunities [56]. Whereas early work on frameworks and design spaces focused on fundamental HCI aspects, such as the classification and taxonomy of input devices [57, 58], later examples formed a valuable basis for development of the corresponding fields, e.g. design spaces for mobile phone input [59], public displays [60] and multimodal 


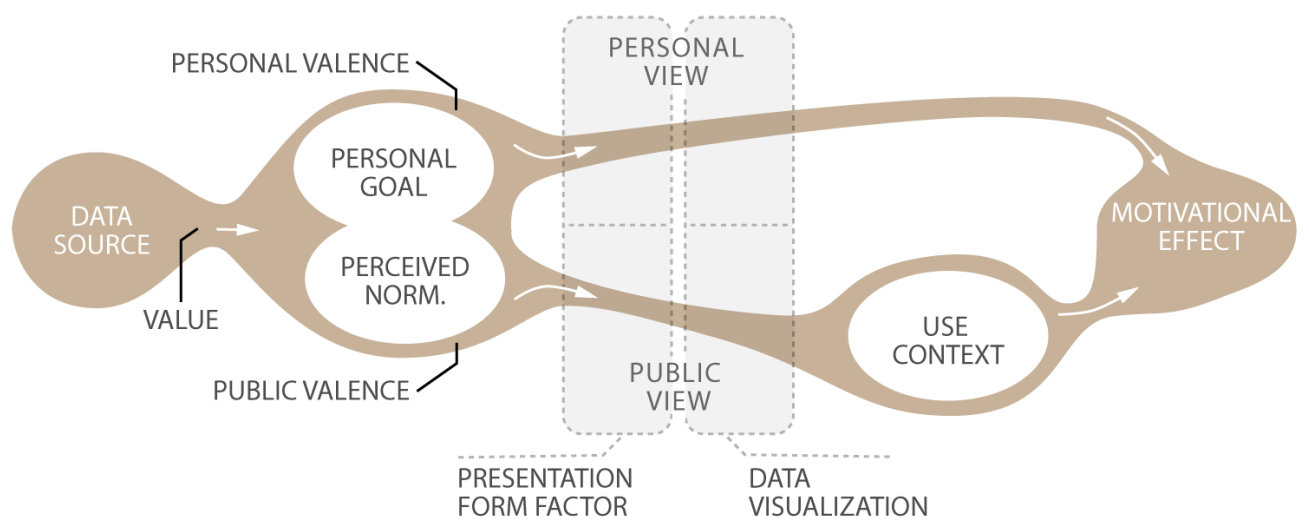

Figure 6: Model for wearable personal and public display of wearable wellness tracker information

interaction [61]. Our aim is that the model presented in this paper provides initial steps towards a structured view to the topic of wearable displays for wellness data presentation and complement other frameworks in the same domain such as the wellness tracker goal framework [7].

In this section we, firstly, introduce and discuss each factor in the model, relating it to our prototypes and study results. Secondly, the following section reflects on the findings from our two user studies against it.

\subsection{Data Source}

Prior work has created wearable display concepts covering a wide variety of different data sources including, steps [22], sleep [52], heart rate [53], body temperature [37] and emotional state [36]. However, prior work has not directly addressed the sensitivity of design and experience to different data sources. In those works that have included multiple data sources, e.g., $[36,37]$, this has not been a focus of the work. We note that not all data sources are the same, being either direct measurements (e.g., temperature), data which is calculated from sensor measurements (e.g., number of steps), or data resulting from more complex algorithms, such as sleep phase identification. However, for all data, perceived accuracy forms a critical element in the experience and impact of use (see Niess \& Woźniak [7] on trust).

Our study participants considered some data sources to be more private than other. For example, many participants felt information related to sleep was more private than information on their level of activity. We speculate 
that lack of sleep is widely understood to be reflected in an individual's work performance, yet the effect of limited activity is less directly observable.

\subsection{Valence}

The value from the data source takes on a positive or negative valence based on the perception of its value [49]. Whilst many of the perception thresholds that define valence are based on medical research, others may be cultural or based on targets created by groups or even individuals. Additionally, we introduce the concept of polarity to handle reporting the same high level parameter from different directions, e.g., daily activity may be reported as number of steps (positive polarity) or the amount of time sitting (negative polarity).

To represent user concerns regarding presenting a positive public image $[24,48]$, our model introduces two data valences that exist side-by-side, namely personal and public valences. The personal valence, i.e. the user's own perception on how good/bad the measured value from the wellness tracker is, is the result of comparing the measurement against the user's personal goals. In contrast, the public valence is the perception of how the value is received by the public, i.e. co-located people with whom the data is shared with through a public display. This is a major difference when designing for a personal wellness display, where data is typically shown in respect to personal goals and activity history.

Our focus group also found that the perception of what was generally regarded as healthy or unhealthy behavior had a strong effect on how the data display was perceived, for example in social situations where they felt pressure to impress those around them there was a preference to convey only positive information about themselves.

\subsection{Presentation Form Factor}

The presentation form factor can take various forms, e.g., embedded in a shirt [8], a hat integrated display [34], or smart bracelet, and data visualization within this form needs to be considered. In our model we divide the output space in two, one for personal view, and one for the eyes of the public. Within this framework concepts can e.g. utilise two separate displays, or a single context adaptive display.

Our study findings also locate well in this model. Wearing a public display increased the importance of the co-located community's role. As the tracker data is shared and observed immediately by surrounding people, feedback 
can be directly obtained. As well as impacting the wearer, the people around the wearer are also affected. For example, when noticing how long they had been sitting, the whole group could decide it was time to have a walk. Whilst it was also commented that this kind of public display functionality could be interpreted as asking for pity, there is potential for it to contribute to social support and work safety [62]). The potential for the display to indicate that the wearer needs support from those around them is an interesting finding, as prior work has identified the wish to offer support [18, 20].

\subsection{Data Visualization}

Prior work presented a huge variety of data visualisation approaches presenting data, e.g., ambiently, numerically, descriptively or graphically, as single values or trends. Here, issues related to the design space of wearable displays (screen size, resolution, and usage context) are relevant factors $[29,60]$.

Some participants preferred abstract, ambient display of tracker data, limiting the information distribution through the need for interpretation. Such approaches address the challenge of displaying negative data values which can be less immediately eye-catching and require the viewer to understand the visual coding used. Glancability was also raised by our participants, which has previously been studied only for smart watch data visualizations [33].

\subsection{Use Context}

The context of use plays a major role in how a public wearable display is perceived and how the user takes the audiences reactions or the social pressure it creates. In contrast, and by definition, the private view of our model is unaffected by context. When sharing tracked data the "where?" and "who with?" are of paramount importance. Sharing context is a highly influential factor both through the pressure on one's public image, but also potentially from the support the user can receive from co-located people.

From our studies we found that context played a central role how wearing a public wellness display was perceived. Rather than the physical context, the social context and the co-located people were perceived most important. This suggests that an interesting direction for future work would be to adapt the data displayed on wearable tracker displays depending on the surrounding people, e.g., friends, family, colleagues or strangers. 


\section{Discussion}

Reflecting our model against works exploring self-presentation from the psychological viewpoint $[63,64,65]$ provides further grounding for our findings. Goffman [63] describes people's actions in social encounters as analogous to actors on a stage, playing roles to fit to the expected social context. With this approach, wearable displays may be seen as props in the show, which, those actors with more extrovert personalities, may wish to engage as part of their presentation to the audience. Focusing on the private vs. public self, Schenkler [64] identifies two axes: motive, i.e. accuracy or personal advantage and audience, i.e. the private or public nature of the activity being performed.

Within this frame, Snyder [65] identifies two categories of individuals: high self-monitors that adapt their behavior to make a favourable impression on others and low self-monitors who are not unduly concerned about opinion of those around them. Thus, our model's separation of the personal and private, and who is able to view the display, well supports the underlying motivations. For Snyder's high self-monitors [65], the public dimensions of our model will have a stronger influence than the private, and vice versa for low self-monitors.

From our evaluations, including both a constrained focus group setting as well as an in-the-wild setting, we conclude that this "hybrid" methodology is valuable to obtain a broader picture of the domain. For example, while participants in the focus group expressed strong concerns for cases where a potentially negative image of them was conveyed, this concern was much less pronounced among people who actually tested the prototype in-the-wild (where only $22 \%$ echoed this concern). Different study paradigms have their strengths when it comes to investigating different research questions. In particular, we found that evaluations in-the-wild are particularly useful if the goal is to test a design regarding user acceptance, social implications, privacy concerns, and user experience. More controlled investigations in the lab being more appropriate to obtain early feedback in the design process and identify interesting directions for future work.

As a limitation, we recognize that our work does not contain a longterm study. This aspect should be investigated to gain further knowledge about the user perceptions of wearable public displays. We also recognise the transferability of our findings is limited by our single culture sample. As noted in our review of related work, attitudes to self-presentation are influenced by culture and gender [26, 27, 25], as well as individual aspects [65]. 
For future researchers and designers, our model provides opportunities to design and build novel wearable displays. As a next step, we plan to further explore the form and content of wearable displays for tracker data, moving more to the direction of aesthetic, clothing design integrated, ambient display. With information presentation aspects, it is of interest to investigate how quickly users can grasp the meaning of an abstract representation of data, and generally study the evaluation methods for wearable displays, as done in other domains of public displays [66]. Further, one interesting aspect raised in our study is the effect of wearer non-visibility of the display.

\section{Conclusions}

Sharing wellness tracker information with selected individuals has been identified as a motivational factor for the adoption of healthy lifestyles. We have presented two functional prototypes - the tracker badge and the hat tracker - which were used as probes in two focus groups and later evaluated them in an in-the-wild study $(n=12)$. Both studies highlighted the positive potential for such concepts. Leveraging findings from related work, we present a model for the design of wearable displays for tracker data. The model distinguishes between personal and public views of wearable display, which may have differing characteristics. Whilst social context is of highest influence, the data source, its valence and the presentation medium are also identified as factors affecting users' willingness to utilize such displays.

\section{Acknowledgements}

This research has been partly supported by the Academy of Finland, TechFashion project and it was partially supported by the European Unions Horizon 2020 Programme under ERCEA grant no. 683008 AMPLIFY.

\section{References}

[1] E. K. Choe, N. B. Lee, B. Lee, W. Pratt, J. A. Kientz, Understanding quantified-selfers' practices in collecting and exploring personal data, in: Proceedings of the 32Nd Annual ACM Conference on Human Factors in Computing Systems, CHI '14, ACM, 2014, pp. 1143-1152. 
[2] N. van Berkel, C. Luo, D. Ferreira, J. Goncalves, V. Kostakos, The curse of quantified-self: An endless quest for answers, in: Adjunct Proceedings of the 2015 ACM International Joint Conference on Pervasive and Ubiquitous Computing and Proceedings of the 2015 ACM International Symposium on Wearable Computers, UbiComp/ISWC'15 Adjunct, ACM, New York, NY, USA, 2015, pp. 973-978.

[3] A. T. Fialho, H. van den Heuvel, Q. Shahab, Q. Liu, L. Li, P. Saini, J. Lacroix, P. Markopoulos, ActiveShare: Sharing challenges to increase physical activities, in: CHI '09 Extended Abstracts on Human Factors in Computing Systems, CHI EA '09, ACM, 2009, pp. 4159-4164.

[4] A. Khalil, S. Abdallah, Harnessing social dynamics through persuasive technology to promote healthier lifestyle 29 (2013) 2674-2681.

[5] S. A. Munson, E. Krupka, C. Richardson, P. Resnick, Effects of public commitments and accountability in a technology-supported physical activity intervention, in: Proceedings of the 33rd Annual ACM Conference on Human Factors in Computing Systems, CHI '15, ACM, 2015, pp. $1135-1144$.

[6] O. Zuckerman, A. Gal-Oz, Deconstructing gamification: Evaluating the effectiveness of continuous measurement, virtual rewards, and social comparison for promoting physical activity, Personal Ubiquitous Comput. 18 (2014) 1705-1719.

[7] J. Niess, P. W. Woźniak, Supporting meaningful personal fitness: The tracker goal evolution model, in: Proceedings of the 2018 CHI Conference on Human Factors in Computing Systems, CHI '18, ACM, New York, NY, USA, 2018, pp. 171:1-171:12.

[8] M. Mauriello, M. Gubbels, J. E. Froehlich, Social fabric fitness: The design and evaluation of wearable e-textile displays to support group running, in: Proceedings of the SIGCHI Conference on Human Factors in Computing Systems, CHI '14, ACM, 2014, pp. 2833-2842.

[9] S. Schneegass, S. Ogando, F. Alt, Using on-body displays for extending the output of wearable devices, in: Proceedings of the 5th ACM International Symposium on Pervasive Displays, PerDis '16, ACM, 2016, pp. $67-74$. 
[10] A. Ahtinen, M. Isomursu, Y. Huhtala, J. Kaasinen, J. Salminen, J. Häkkilä, Tracking outdoor sports - user experience perspective, in: E. Aarts, J. L. Crowley, B. de Ruyter, H. Gerhäuser, A. Pflaum, J. Schmidt, R. Wichert (Eds.), Ambient Intelligence, Springer Berlin Heidelberg, Berlin, Heidelberg, 2008, pp. 192-209.

[11] S. Asimakopoulos, G. Asimakopoulos, F. Spillers, Motivation and user engagement in fitness tracking: Heuristics for mobile healthcare wearables, in: Informatics, volume 4, Multidisciplinary Digital Publishing Institute, p. 5.

[12] J. Kim, Y.-W. Park, T.-J. Nam, BreathingFrame: An inflatable frame for remote breath signal sharing, in: Proceedings of the Ninth International Conference on Tangible, Embedded, and Embodied Interaction, TEI '15, ACM, 2015, pp. 109-112.

[13] H. C. Min, T.-J. Nam, Biosignal sharing for affective connectedness, in: CHI '14 Extended Abstracts on Human Factors in Computing Systems, CHI EA '14, ACM, 2014, pp. 2191-2196.

[14] P. Slovak, J. Janssen, G. Fitzpatrick, Understanding heart rate sharing: Towards unpacking physiosocial space, in: Proceedings of the SIGCHI Conference on Human Factors in Computing Systems, CHI '12, ACM, 2012, pp. 859-868.

[15] J. Werner, R. Wettach, E. Hornecker, United-pulse: Feeling your partner's pulse, in: Proceedings of the 10th International Conference on Human Computer Interaction with Mobile Devices and Services, MobileHCI '08, ACM, New York, NY, USA, 2008, pp. 535-538.

[16] A. Fedosov, E. Mencarini, P. Woźniak, K. Knaving, M. Langheinrich, Towards understanding digital sharing practices in outdoor sports, in: Proceedings of the 2016 ACM International Joint Conference on Pervasive and Ubiquitous Computing: Adjunct, UbiComp '16, ACM, New York, NY, USA, 2016, pp. 861-866.

[17] P. W. Wozniak, A. Fedosov, E. Mencarini, K. Knaving, Soil, rock, and snow: On designing for information sharing in outdoor sports, in: Proceedings of the 2017 Conference on Designing Interactive Systems, DIS '17, ACM, New York, NY, USA, 2017, pp. 611-623. 
[18] T. Fritz, E. M. Huang, G. C. Murphy, T. Zimmermann, Persuasive technology in the real world: a study of long-term use of activity sensing devices for fitness, in: Proceedings of the SIGCHI Conference on Human Factors in Computing Systems, ACM, pp. 487-496.

[19] K. Park, I. Weber, M. Cha, C. Lee, Persistent sharing of fitness app status on twitter, in: Proceedings of the 19th ACM Conference on Computer-Supported Cooperative Work \& Social Computing, CSCW '16, ACM, New York, NY, USA, 2016, pp. 184-194.

[20] S. Lomborg, K. Frandsen, Self-tracking as communication, Information, Communication \& Society 19 (2016) 1015-1027.

[21] B. Choi, Z. J. Jiang, Encouraging active lifestyle with social sharing: A study on mobile fitness app (2018).

[22] A. Morrison, V. Bakayov, Stickers for steps: a study of an activity tracking system with face-to-face social engagement, Proceedings of the ACM on Human-Computer Interaction 1 (2017).

[23] S. Consolvo, K. Everitt, I. Smith, J. A. Landay, Design requirements for technologies that encourage physical activity, in: Proceedings of the SIGCHI conference on Human Factors in computing systems, ACM, pp. 457-466.

[24] X. Gui, Y. Chen, C. Caldeira, D. Xiao, Y. Chen, When fitness meets social networks: Investigating fitness tracking and social practices on werun, in: Proceedings of the 2017 CHI Conference on Human Factors in Computing Systems, CHI '17, ACM, New York, NY, USA, 2017, pp. $1647-1659$.

[25] L. Wang, P.-L. P. Rau, G. Salvendy, A cross-culture study on older adults' information technology acceptance, International Journal of Mobile Communications 9 (2011) 421-440.

[26] S. Duval, H. Hashizume, Perception of wearable computers for everyday life by the general public: impact of culture and gender on technology, in: International Conference on Embedded And Ubiquitous Computing, Springer, pp. 826-835. 
[27] Y. Li, X. Wang, X. Lin, M. Hajli, Seeking and sharing health information on social media: A net valence model and cross-cultural comparison, Technological Forecasting and Social Change 126 (2018) 28-40.

[28] P. C. Shih, K. Han, E. S. Poole, M. B. Rosson, J. M. Carroll, Use and adoption challenges of wearable activity trackers (2015).

[29] S. Schneegass, T. Olsson, S. Mayer, K. van Laerhoven, Mobile interactions augmented by wearable computing: A design space and vision 8 (2016) 104-114.

[30] J. Falk, S. Björk, The bubblebadge: A wearable public display, in: CHI '99 Extended Abstracts on Human Factors in Computing Systems, CHI EA '99, ACM, New York, NY, USA, 1999, pp. 318-319.

[31] S. A. Lewis, G. D. Havey, B. Hanzal, Handheld and bodyworn graphical displays: presented at the international symposium on wearable computing (ISWC'98), in: Digest of Papers. Second International Symposium on Wearable Computers (Cat. No.98EX215), pp. 102-107.

[32] A. Colley, M. Pakanen, S. Koskinen, K. Mikkonen, J. Häkkilä, Smart handbag as a wearable public display - exploring concepts and user perceptions, in: Proceedings of the 7th Augmented Human International Conference 2016, AH '16, ACM, New York, NY, USA, 2016, pp. 7:1-7:8.

[33] R. Gouveia, F. Pereira, E. Karapanos, S. A. Munson, M. Hassenzahl, Exploring the design space of glanceable feedback for physical activity trackers, in: Proceedings of the 2016 ACM International Joint Conference on Pervasive and Ubiquitous Computing, ACM, pp. 144-155.

[34] W. Walmink, D. Wilde, F. F. Mueller, Displaying heart rate data on a bicycle helmet to support social exertion experiences, in: Proceedings of the 8th International Conference on Tangible, Embedded and Embodied Interaction, TEI '14, ACM, New York, NY, USA, 2013, pp. 97-104.

[35] P. Burns, C. Lueg, S. Berkovsky, Activmon: Encouraging physical activity through ambient social awareness, in: CHI '12 Extended Abstracts on Human Factors in Computing Systems, CHI EA '12, ACM, 2012, pp. 2363-2368. 
[36] M. A. Williams, A. Roseway, C. O’Dowd, M. Czerwinski, M. R. Morris, Swarm: An actuated wearable for mediating affect, in: Proceedings of the Ninth International Conference on Tangible, Embedded, and Embodied Interaction, TEI '15, ACM, New York, NY, USA, 2015, pp. 293-300.

[37] R. Ashford, Responsive and emotive wearables: Devices, bodies, data and communication, in: Proceedings of the 2014 ACM International Symposium on Wearable Computers: Adjunct Program, ISWC '14 Adjunct, ACM, New York, NY, USA, 2014, pp. 99-104.

[38] M. Altmeyer, P. Lessel, T. Sander, A. Krüger, Extending a gamified mobile app with a public display to encourage walking, in: Proceedings of the 22Nd International Academic Mindtrek Conference, Mindtrek '18, ACM, New York, NY, USA, 2018, pp. 20-29.

[39] R. A. Khot, F. F. Mueller, Sweat-atoms: Turning physical exercise into physical objects, in: CHI '13 Extended Abstracts on Human Factors in Computing Systems, CHI EA '13, ACM, 2013, pp. 3075-3078.

[40] S. Stusak, A. Tabard, F. Sauka, R. A. Khot, A. Butz, Activity sculptures: Exploring the impact of physical visualizations on running activity, IEEE Transactions on Visualization and Computer Graphics 20 (2014) 2201-2210.

[41] E. Arroyo, L. Bonanni, N. Valkanova, Embedded interaction in a water fountain for motivating behavior change in public space, in: Proceedings of the SIGCHI Conference on Human Factors in Computing Systems, CHI '12, ACM, New York, NY, USA, 2012, pp. 685-688.

[42] R. A. Khot, J. Lee, D. Aggarwal, L. Hjorth, F. F. Mueller, TastyBeats: Designing palatable representations of physical activity, in: Proceedings of the 33rd Annual ACM Conference on Human Factors in Computing Systems, CHI '15, ACM, 2015, pp. 2933-2942.

[43] C. Fan, J. Forlizzi, A. K. Dey, A spark of activity: Exploring informative art as visualization for physical activity, in: Proceedings of the 2012 ACM Conference on Ubiquitous Computing, UbiComp '12, ACM, 2012, pp. 81-84. 
[44] K. Davis, E. Owusu, L. Marcenaro, L. Feijs, C. Regazzoni, J. Hu, Evaluating human activity-based ambient lighting displays for effective peripheral communication, in: Proceedings of the 11th EAI International Conference on Body Area Networks, BodyNets '16, ICST (Institute for Computer Sciences, Social-Informatics and Telecommunications Engineering), 2016, pp. 148-154.

[45] T. Nakajima, V. Lehdonvirta, Designing motivation using persuasive ambient mirrors 17 (2013) 107-126.

[46] J. Fortmann, T. C. Stratmann, S. Boll, B. Poppinga, W. Heuten, Make me move at work! an ambient light display to increase physical activity, in: Proceedings of the 7th International Conference on Pervasive Computing Technologies for Healthcare, PervasiveHealth '13, ICST (Institute for Computer Sciences, Social-Informatics and Telecommunications Engineering), 2013, pp. 274-277.

[47] Y. Rogers, W. R. Hazlewood, P. Marshall, N. Dalton, S. Hertrich, Ambient influence: Can twinkly lights lure and abstract representations trigger behavioral change?, in: Proceedings of the 12th ACM International Conference on Ubiquitous Computing, UbiComp '10, ACM, 2010, pp. 261-270.

[48] M. W. Newman, D. Lauterbach, S. A. Munson, P. Resnick, M. E. Morris, It's not that i don't have problems, i'm just not putting them on facebook: challenges and opportunities in using online social networks for health, in: Proceedings of the ACM 2011 conference on Computer supported cooperative work, ACM, pp. 341-350.

[49] M. Hassib, M. Khamis, S. Schneegass, A. S. Shirazi, F. Alt, Investigating user needs for bio-sensing and affective wearables, in: Proceedings of the 2016 CHI Conference Extended Abstracts on Human Factors in Computing Systems, CHI EA '16, ACM, 2016, pp. 1415-1422.

[50] S. Consolvo, P. Klasnja, D. W. McDonald, D. Avrahami, J. Froehlich, L. LeGrand, R. Libby, K. Mosher, J. A. Landay, Flowers or a robot army?: Encouraging awareness \& activity with personal, mobile displays, in: Proceedings of the 10th International Conference on Ubiquitous Computing, UbiComp '08, ACM, 2008, pp. 54-63. 
[51] A. Prasad, J. Sorber, T. Stablein, D. Anthony, D. Kotz, Understanding sharing preferences and behavior for mhealth devices, in: Proceedings of the 2012 ACM workshop on Privacy in the electronic society, ACM, pp. $117-128$.

[52] A. Sahami Shirazi, J. Clawson, Y. Hassanpour, M. J. Tourian, A. Schmidt, E. H. Chi, M. Borazio, K. Van Laerhoven, Already up? using mobile phones to track \& share sleep behavior 71 (2013) 878-888.

[53] M. Hassib, D. Buschek, P. Wozniak, F. Alt, HeartChat: Heart rate augmented mobile chat to support empathy and awareness, in: Proceedings of the 2017 CHI Conference on Human Factors in Computing Systems, CHI '17, ACM, 2017, pp. 2239-2251.

[54] M. L. Jacobs, J. Clawson, E. D. Mynatt, Comparing health information sharing preferences of cancer patients, doctors, and navigators, in: Proceedings of the 18th ACM Conference on Computer Supported Cooperative Work \& Social Computing, CSCW'15, ACM, 2015, pp. 808-818.

[55] R. Rettner, The truth about '10,000 steps' a day, ????

[56] S. K. Card, J. Mackinlay, The structure of the information visualization design space, in: Proceedings of the IEEE Symposium on Information Visualization 1997, pp. 92-99.

[57] W. Buxton, Lexical and pragmatic considerations of input structures 17 (1983) 31-37.

[58] S. K. Card, J. D. Mackinlay, G. G. Robertson, A morphological analysis of the design space of input devices, ACM Trans. Inf. Syst. 9 (1991) 99122 .

[59] R. Ballagas, M. Rohs, J. G. Sheridan, J. Borchers, The design space of ubiquitous mobile input, in: Ubiquitous and Pervasive Computing: Concepts, Methodologies, Tools, and Applications, IGI Global, 2010, pp. 439-461.

[60] J. Müller, F. Alt, D. Michelis, A. Schmidt, Requirements and design space for interactive public displays, in: Proceedings of the 18th ACM International Conference on Multimedia, MM '10, ACM, New York, NY, USA, 2010, pp. 1285-1294. 
[61] L. Nigay, J. Coutaz, A design space for multimodal systems: Concurrent processing and data fusion, in: Proceedings of the INTERACT '93 and CHI '93 Conference on Human Factors in Computing Systems, CHI '93, ACM, New York, NY, USA, 1993, pp. 172-178.

[62] D. MacLean, A. Roseway, M. Czerwinski, MoodWings: A wearable biofeedback device for real-time stress intervention, in: Proceedings of the 6th International Conference on PErvasive Technologies Related to Assistive Environments, PETRA '13, ACM, 2013, pp. 66:1-66:8.

[63] E. Goffman, The presentation of self in everyday life, Anchor Books, 1959.

[64] B. R. Schlenker, Self-identification: Toward an integration of the private and public self, in: Public self and private self, Springer, 1986, pp. 21-62.

[65] M. Snyder, Public appearances, Private realities: The psychology of selfmonitoring., WH Freeman/Times Books/Henry Holt \& Co, 1987.

[66] F. Alt, S. Schneegaß, A. Schmidt, J. Müller, N. Memarovic, How to evaluate public displays, in: Proceedings of the 2012 International Symposium on Pervasive Displays, PerDis '12, ACM, New York, NY, USA, 2012, pp. 17:1-17:6. 